\title{
The Operating Obstacles and Solving Strategy of Translational Medicine in China
}

\author{
Hai Jiang and Yin-He Cui
}

\begin{abstract}
The essence of translational medicine is the collaborative innovation of scientific research management. By investigating, there are the operation obstacles in five aspects about translational medicine in China, such as subject language, thinking mode, the top-level design, system and mechanism, organizational culture. Therefore, translational medicine needs to integrate the entire process by the common goal, takes the joint efforts to complete research projects by the research mode of "big science", focuses on the interests of every research personnel, promote scientific research resources flow freely, evaluates the scientific achievements reasonably, and eventually realizes the effective operation of scientific research complex networks of multi-agent collaborative in translational medicine.
\end{abstract}

Index Terms-Translational medicine, research mode, operating obstacles, solving strategy.

\section{INTRODUCTION}

As a new concept of medical scientific research, Translational Medicine introduces clinical medical practice into laboratory research and applies obtained research achievements into practical application so as to realize the double-way knowledge transformation [1]. Chen Zhu, as former Minister of Health, has pointed out that translational medicine is not a new discipline, but a scientific research method, a way of thinking and a research organization mechanism [2].

A great many of experts and scholars put forward that the construction of translational medicine mode needs the combination of multidisciplinary background knowledge [3]. In the meanwhile, it is of great significance to eliminate internal obstacles and solve external problems so as to ensure the smooth running of translational medicine.

\section{The TheORETICAL Dilemma of TRANSLATIONAL MEDICINE IN CHINA}

\section{A. The Development of Translational Medicine Research}

As a new model of biomedical development and scientific management, Translation medicine is new leap for medical

Manuscript received March 14, 2016; revised September 25, 2016. This work was supported by the Liaoning Province Science of Education "12TH FIVE-YEAR PLAN" Grant(JG15DB103), Dalian Medical University Teaching Reform Research Council Grant (DYLX15057), and Liaoning Province Academic Library Research Council Grant(L2015001).

Hai Jiang is with Humanities and Social Sciences, Dalian University of Technology (DUT), Faculty of Library, Dalian Medical University (DMU), China (e-mail: jerrylib@163.com).

Yin-He Cui is with Faculty of Humanities and Social Sciences, Dalian University of Technology (DUT), China (e-mail: 565356289@qq.com). development. From obtained literature retrieve, Mckinney GR first put forward the concept of "From Bench to Bedside" in 1966, and lay great foundation of strengthening basis research to clinic application research. In 1992 , Choi DW restate the concept of "Bench to Bedside" which exert great influence in medical profession. The concept of Translation Research is formally put forward in 1994. The definition of Translation Medicine first arisen in Lancet in 1996. The United States of National Institutes of Health (NIH) published NIH Roadmap in 2003 proposed that Translation Medicine research would be the determined direction and core content of future medical research and construction. At present, The Translation Medicine has become a forefront issue in biomedical fields [4]-[7].

Translation Medicine adopts the holistic thinking to explore life regulation, and opens " $4 \mathrm{P}$ " medical model by systematic control strategy method: Predictive, Preventive, Personalized and Participatory. "4P-mode" lays great emphasis on human life and the mutual influence of human life and diseases, through appropriate guidance of human behavior to prevent diseases, improve higher quality of human life [8]. The cohesion of life science, biotechnology and related modern science contributes a lot to the transformation of medical system in diagnosis of diseases, early intervention and further treatment so as to comprehensively improve the quality of human life and decrease medical cost.

\section{B. The Theoretical Dilemma of Translational Medicine in China}

NIH formally discussed the concept of Translation Medicine when it gave the explanation of NIH Roadmap in 2003 and made strategic planning for health system construction and development in the United States[9].The target of Roadmap mainly concentrated in three areas: exploring new method, building scientific research team, designing clinical research enterprise. The targets include 28 projects which involves systematic biology, molecular libraries and molecular imaging, structural biology, bioinformatics and computational biology, the establishment of medicine, cross platform and cross talented person's raise, cross team construction, accelerating the transformation of basic research to clinical. At the same time, the translational medicine develops rapidly in China and 129 translational medicine research centers and platforms has been set up from January 2009 to the end of December of 2013 [10]. The number of translational medicine has made great breakthrough, but system innovation and content construction are still deficient.

Till now, there has been uniform understanding of "What is Translation Medicine" in China and researches on 
Translation Medicine are mainly confined to clinic researchers. From the perspective of literature analysis, most achievements are in the stage of theoretical introduction, clinical practice, preventive medicine, laboratory medicine and pharmacology and other branches of specific applications Whereas practical problems in the process of translational medicine are few and far between, which includes how to deepen medical philosophy on Translational Medicine, the construction of demonstration mode, how to construct fund-raising channels and how to evaluate performance appraisal? All these issues hinder the improvements of Translational Medicine and become the bottleneck of further research. So this paper attempts to dig out these problems and analysis these obstacles in a better way [11].

\section{THE ANALYSIS TO OPERATION BARRIER OF TRANSLATIONAL MEDICINE}

In the process of constructing Translational Medicine Mode, we need multidisciplinary knowledge background of scientific research and cross fusion collaboration system. The function of the system itself is a complex scientific research network which causes the problems of subject language, thinking mode, top-level design, mechanism of different systems, and organization culture.

\section{A. The Differences of Subject Language}

Whether breakthroughs will be made in future biomedical depends on collaboration with physics, chemistry, materials science, information technology, engineering and other multi-disciplines both at home and abroad. However, modern biological medicine has evolved into a complex system with the guidance of reductionism and each discipline has its own domain, different research paradigm of problem solving, which leads to their unique academic language in describing same problem. In the meanwhile, misunderstanding or misuse will cause understanding difficulty. So, how to guide the transformation of medical scientific research from multiple levels has become a big issue.

\section{B. The Obstacles of Thinking Mode}

Thinking mode refers to cognitive processes, research methods and rules of behaviors, which is centralized coding, retrieval function, integration. Scientific research teams that belong to different disciplines have fixed thinking mode and principles. From the perspective of scientific research, translational medicine is embodied "big science" and system theory, which radically prevents the problems of dividing smaller domains. However, cognitive differences have become more and more obvious in the process of applying basic research into clinical practice due to various thinking modes. It has been hard to integrate other research groups with the penetration to specific stages.

\section{The Lacking of Top-Level Design}

However, constructing translation medicine has the defect of lacking of top-level design and the fully consideration of collaborative relationships. In China, lacking of sharing cooperation is becoming more and more outstanding in advancing translational medicine center. Institutions of translational medicine has become a virtual subject, in the meanwhile, platform cannot achieve technological resource sharing among scientific research teams. Translational medicine aims to access resources and redistribute resources, which cannot produce cluster effect. So the decline of overall efficiency decides the evitable difficulty in cultivating innovative talents.

\section{The Obstacles of System and Mechanism}

Present technology mechanism restricts the development of translational medicine. First of all, researchers mainly confined in limited areas which lack of closed collaboration with other institutions. Such kind of segmentation confines translational medicine in relatively small scale and affects further development. Second, both national and local translational medicine centers rely too much on established research centers and sharing resources from others, as a result, the rate of using research infrastructure and equipment is low. Third, the National Science Fund (NSF) set up by division and subjects in China, so the research subject of translational medicine must determine subject category. These series issues should be paid more attention in project bidding, investment fund, subject setting, reward and evaluation system in clinic practice and research of public health.

\section{E. The Differences of Organizational Culture}

Different research teams of translational medicine will develop their inherent values and it will strongly exert influence on what and how to do researches. When team's basic values contradict with research question or selected information, members of team will be more difficult to integrate. On the other hand, present scientific research performance evaluation mechanism attaches great importance of individual abilities rather than the whole team's collaboration. The evaluation of classification and different responsibilities are also neglected, and all these reasons lead to inactive collaborative atmosphere in the process. What's more Some scientific researchers are unwilling to share their achievements with others in order to maintain their own academic status, so the team's overall scientific research efficiency will decrease greatly.

\section{THE SOlVING StRATEGy TO OPERATION OBSTACLES OF Translational MEDicine RESEARCH ModE}

Through analyzing obstacles of translational medicine, we put forward the corresponding solution strategy from various angles and form the pattern of translational medicine. The concrete content includes the following five aspects:

\section{A. The Common Target Integrate the Whole Translational Medicine Process}

The core value of translational medicine is realizing basic research, clinical application, public health. In order to make more efficient mode of translational medicine, mutual integration of research network must be guaranteed in order to promote background knowledge of different disciplines. Scientific researchers could reveal pathogenesis to help laboratory understand human body through clinic observation. With the combination of both short and long term goal, 
translational medicine will speed up its development. The long-term goal is to promote the development of translational medicine industry and the short-term goal is to concise scientific problems from demand of clinical.

\section{B. The Pattern of "Big Science"}

The mode of translational medicine is a complex network with the characteristic of interdisciplinary, cross-industry, multi-agent collaboration. With the rapid development of biomedical domain, predicting medicine frontier and grasping medicine development cannot be undertaken by someone or a certain way. The revolution of scientific research management system needs "big science" research model and the procedure of interdisciplinary research team to satisfy the needs of national economic and social development strategies. It benefits evaluation mechanism and personnel flow regular by adopting big science mode.

\section{Focus on the Interests of Each Researcher}

Translational medicine is essentially a complex network and every scientific research person is a network node. The sustainable development of network benefits not only research organization but also each scientific personnel based on their achievements. Translational medicine research model will succeed on condition that each scientific research personnel of interests and responsibilities are clear because the entire research collaboration network is composed of individual efforts. The ultimate achievements require every researcher's efforts from problems finding, process execution and testing. If there is no clear evaluation mechanism, the outcome of translational medicine research model will lose its power, therefore, establishing incentive mechanism is of great necessity and it will make the whole transformation process dynamic.

\section{Promote the Flow of Scientific Research Resources Freely}

Translational medicine is the embodiment of modern medical model system with the characteristic of continuity, open practical system and mutual ways. The reconstruction of translational medicine needs knowledge innovation, technology resources promotion and fully utilization of technology platforms. The biggest challenge is that it contains more institutions and entities than general research network in order to ensure the fluent flow of scientific research achievements and technology resources. In the process of constructing Translational Medicine Database, it is of great necessity for scientific research subjects to understand and respect each other and make the maximization of resource utilization in order to realize resources sharing.

\section{E. The Evaluation of Translational Medicine Scientific Research}

Translational medicine enters a new field of complexity research which not only needs the cooperation of biologist but also depends on multi-disciplinary fusion of chemical, information technology and mathematics. In the process of assessing scientific value, the whole evaluation system should be comprehensive in order to arouse enthusiasm of scientific personnel and evoke their creativity. Strengthening infiltration among different disciplines is another principle to construct Translational Medicine model with the goal of populating application.

\section{The Design of Translational Medicine Research MECHANISM WITH THE PERSPECTIVE OF NEW INSTITUTIONAL ECONOMICS}

From the perspective of New Institutional Economics translational medicine research system, it is in great need to solve the problem of concept, management and technology. Therefore, the mechanism design needs to be carried out from the following aspects through standardized system design.

\section{A. Trust Building Mechanism}

New institutional economics thinks that the rise of costs between main body is caused by inadequate trust mechanism, and the trust between the two sides can reduce transaction cost and promote cooperation greatly. Translational Medicine proposes the harmonious cooperative relationship, and confront the cost of sharing scientific research, the core technology protection and intellectual property issues. All these problems hinder the persistence of collaboration network. New institutional economics requires establishing trust mechanism between translational medicine subjects through system design and ensuring the freedom of scientific research personnel. Therefore, the establishment of trusting mechanism is the basic requirement of translational medicine research mode.

\section{B. Coordination Mechanism}

The structure of existing scientific research management organization is the pyramid of hierarchical structure. The long chains determines the difficulty in communication, which leads to the overall research work efficiency is low and management cost is high. The network of translation medicine research with the perspective of new institutional economics is by contract. Each node enters into a contract which guarantees stakeholder responsibility, right and benefit, resource deployment and use. This kind of contract coordination mechanism of translational medicine needs a longer commitment and agreement between subjects in order to ensure the effective operation of the whole research collaboration model, as well as for scientific research task to share the benefits and risks.

\section{Allocation Mechanism}

"Potential" violence was proposed by North in Distribution theory which put forward that the state processes comparative advantage in terms of violence, if it can take between citizens of equal distribution, can produce contractual countries. Translational medicine research model is an open system structure of collaborative relationship between members based on common interests, different members in translational medicine research network resources and the risk is different. Research network allocation mechanism in accordance with the principle of "responsibility - peer interests" in property ownership interest distribution will manifest in system design. At the same time, the allocation mechanism not only needs the consideration of physical inputs to the system design, but also needs to take the intangible investment into account, because scientific research cooperation between many members of the network 
is internalized in translational medicine research modes.

\section{Constraint Mechanism}

The new economic system learning hypothesis emphasizes the profit to motive people. On this basis, the new institutional economics first about system is defined as an individual to self-imposed constraints, and its basic method is game theory. Maximize the efficiency through standard cooperation mechanism to solve the contradiction between the players. Translational medicine research system needs the constraints between subjects, economic and social responsibility are confined by institutional constraint. The specific method of cooperation and division of tasks also need to be decided by constraint so as to work as implementation of post responsibility system, strengthen the project management. At the same time, the constraint mechanism of the translational medicine research model is the part between the main body behavior constraints, constraint range also has certain limits.

\section{E. Feedback Mechanism}

New institutional economics thinks that path dependence is used to describe the technological changes in the process of self-accumulation and self-reinforcement. Describing the influence of past performance on present and the future by feedback mechanism is similar to the "law of inertia" in physics. Translational medicine research's feedback mechanism design needs to consider the research of cognitive differences between members of the network. The core ability of integration is a gradual process which can solve existing problems in the process of collaboration. But that there are two possibilities: one is that the research part of the members of the network can't meet the requirements of translational medicine scientific research system of indicators, members of restructuring again, even more options. Second, translational medicine research network structural and systematic defects need to overall systematic structure adjustment.

\section{F. Risk Prevention Mechanism}

New institutional economist Williams thought that there is asymmetric information situation in realistic society. Individual behavior will become more complex because of the pursuit of self-interest. Paying too much attention to individual interests and ignoring the interests of others will cause great risk. Each subject in Translational medicine research pattern was independent from each other, there's a benefit sharing according to incomplete contract model implementation research collaboration. But it is also of great necessity of building risk prevention mechanism in order to prevent some potential risks. In the process of mechanism design, first of all, different subjects should recognize the risk in the scientific research cooperation objectively, and try to avoid or even reduce the risk consciously. Secondly, risk prevention mechanism needs to protect the core of each collaboration, subject knowledge and resources, which promotes the main body of translational medicine research network and guarantees the stability of cooperation.

\section{CONCLUSION}

Translational medicine is a complicated engineering system which involves so many uncertain factors. Removing operating obstacles can not only realize conversion of scientific research to clinical stage but also promote efficiency of cooperation network. Solving these confronting problems need conversion of concept, advanced technology and management level with the ultimate goal of realizing promotion of health level in China.

\section{ACKNOWLEDGMENT}

The project is supported by the Liaoning Province Science of Education "12TH FIVE-YEAR PLAN" Grant (JG15DB103), Dalian Medical University Teaching Reform Research Council Grant (DYLX15057), and Liaoning Province Academic Library Research Council Grant (L2015001).

\section{REFERENCES}

[1] F. M. Marincola, "Translational medicine: A two-way road," J Transl Med, vol. 1, p. 1, Jul. 242003.

[2] Z. Chen. (February 2011). Promoting the development of translational medicine, cope with the challenge of the people's health. [Online]. Available: http://www.cas.cn/ys/ysjy/201102/t20110214_3071055.shtml

[3] K. J. Dai, Concept, Policy and Practice of Translational Medicine, 1 China: The Fourth Military Medical University Press, 2012, pp. 3-35.

[4] G. R. McKinney and H. E. Stavely, "From bench to bedside: The biologist in drug development," BioScience, vol. 16, pp. 683-687, October 1, 1966.

[5] D. W. Choi, "Bench to bedside - The glutamate connection," Science, vol. 258, pp. 241-243, Oct. 9, 1992.

[6] J. Geraghty, "Adenomatous polyposis coli and translational medicine," Lancet, vol. 348, pp. 422-422, 1996.

[7] E. Zerhouni, "The NIH roadmap," Science, vol. 302, pp. 63-+, Oct. 3 2003.

[8] Q. Yao, J. Chen, P.-H. Lyu, S.-J. Zhang, F.-C. Ma, and J.-G. Fang, "Knowledge map of artemisinin research in SCI and Medline database," Journal of Vector Borne Diseases, vol. 49, pp. 205-216, Dec 2012.

[9] NIH. National Center for Advancing Translational Sciences (20122013 REPORT). [Online]. Available: http://www.ncats.nih.gov/files/NCATS_2012-2013_Report.pdf

[10] P. B. Fontanarosa and C. D. DeAngelis, "Basic science and translational research in JAMA," JAMA: The journal of the American Medical Association, vol. 287, pp. 1728-1728, 2002.

[11] D. Dougherty and P. H. Conway, "The "3T's" road map to transform US health care - The "how" of high-quality care," Jama-Journal of the American Medical Association, vol. 299, pp. 2319-2321, May 212008.

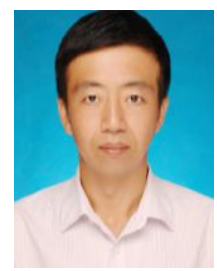

Hai Jiang was born in November, 1978 in Dalian, China. He studied information science in Dalian University of Technology from 1997-2001. Then he received his master degree from Liaoning Normal University in 2008. As a librarian in Dalian Medical University Library from 2008, he is in charge of library management, information management, medical information analysis, information literacy teaching for medical students. He is currently working towards a PhD degree in Dalian University of Technology with the major of science of science and management of S.\& T

He previous researched in information resources sharing and the construction of video teaching resource platform. His research interests are focused on library management, medical information analysis and information literacy education.

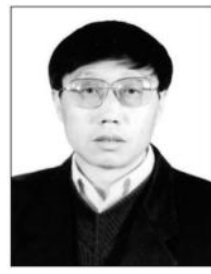

Yin-he Cui is a Univ. Prof in Dalian University of Technology. He was born in 1962 in Haicheng, Liaoning, China. He studied science of science and management of S\&T and information spreading 